\section{HOSPITALIZAÇÕES POR INFECÇÃO RESPIRATÓRIA ASSOCIADA A FATORES AMBIENTAIS}

\author{
Hospitalizations for respiratory infections associated with \\ environmental factors
}
Ingresos por infección respiratoria asociada a factores ambientales

\section{RESUMO}

Objetivo: Avaliar a relação entre variáveis meteorológicas e doenças respiratórias por faixa etária. Métodos: Trata-se de um estudo ecológico de séries temporais para o período compreendido entre 2008 e 2011. Primeiramente, as internações foram analisadas por grupo de faixa etária e, em seguida, relacionadas com informações climáticas, temperatura, precipitação, umidade relativa do ar e velocidade dos ventos, através da climatologia dinâmica e de parâmetros estatísticos, utilizando análise de regressão múltipla, teste qui-quadrado e distribuição normal. Resultados: Os resultados mostraram 12.067 registros de internações por doenças respiratórias e por faixa etária. As maiores médias de internação ocorreram na faixa etária de 1 a 4 anos, com média de 42,09\%, (5.079) e acima de 60 anos, com média de $27,14 \%$ (3.274) para o período de estudo, sendo as pneumonias predominantes, com $81,0 \%$ (9.774) das internações. Verificou-se menor número de internações em janeiro e maior, em junho e julho. Conclusão: Com base nas investigações realizadas, observou-se correlação significativa entre os parâmetros climáticos e as internações por doenças respiratórias.

Descritores: Clima; Saúde; Doenças Respiratórias; Temperatura Ambiente.

\section{ABSTRACT}

Objective: To evaluate the relationship between the meteorological variables and respiratory diseases by age. Methods: This is an ecological time-series study, for the period comprised between 2008 and 2011. First, admissions were analyzed by age group and then related to climate information: temperature, precipitation, relative air humidity, and wind speed, through dynamic climatology and statistical parameters using multiple regression analysis, chi-square test and normal distribution. Results: The results showed 12,067 records of hospital admissions for respiratory diseases according to the age group. The highest average number of hospitalizations was found in the age range from 1 to 4 years, with a mean of $42.09 \%$ (5079), and over 60 years, with a mean of 27.14\% (3274) for the study period. The highest prevalence was due to pneumonia, accounting for $81.0 \%(9,774)$ of admissions. The number of hospitalizations was lower in January and higher in June and July. Conclusion: Based on the investigations carried out, a significant correlation was observed between climate parameters and hospitalization for respiratory diseases.

Descriptors: Climate; Health; Respiratory Tract Diseases; Temperature.

\section{RESUMEN}

Objetivo: Evaluar la relación entre variables meteorológicas y enfermedades respiratorias por franja de edad. Métodos: Se trata de un estudio ecológico de series temporales en el periodo entre 2008 y 2011. En primer lugar, los ingresos fueron analizados por franja de edad y, en seguida, relacionados con las informaciones climáticas, temperatura, precipitación, humidad relativa del aire y la velocidad de los vientos a través de la climatología dinámica y parámetros estadísticos utilizándose del análisis de regresión múltiple, prueba del Chicuadrado y distribución normal. Resultados: Los resultados señalaron 12.067 registros de
Artigo Original
1) Universidade Federal de Mato Grosso do Sul - UFMS - Campo Grande (MS) - Brasil

2) Chinese Center for Disease Control and Prevention - Beijing 102206 - People's Republic of China

3) University of Queensland - Herston QLD - Australia

4) College of Health Sciences, the Public Authority for Applied Education and Training - Kuwait
Recebido em: 05/07/2013 Revisado em: 11/09/2013 Aceito em: 11/08/2014 
ingresos por enfermedades respiratorias y franja de edad. Las mayores medias de ingreso se dieron en la franja de edad de 1 a 4 años con media del 42,09\%, (5.079) y por encima de los 60 años con media del 27,14\% (3.274) en el periodo del estudio, siendo las neumonías las más predominantes en el 81,0\% (9.774) de los ingresos. Se verifico menos ingresos en enero y más en junio y julio. Conclusión: Basado en las investigaciones realizadas, se observó correlación significante entre los parámetros climáticos y los ingresos por enfermedades respiratorias.

Descriptores: Clima; Salud; Enfermedades Respiratorias; Temperatura Ambiental.

\section{INTRODUÇÃO}

Uma compreensão da natureza dos efeitos das condições meteorológicas para a saúde é essencial para a sua proteção. A relação tempo-saúde é associada entre a temperatura e a morbidade/mortalidade. Relações não lineares (U, J ou em V) de temperatura e de morbidade/ mortalidade são observadas ${ }^{(1)}$. Além da temperatura, as variáveis meteorológicas, como umidade, pressão barométrica e precipitação, também são encontradas para serem relacionadas à morbidade/mortalidade ${ }^{(2)}$. No entanto, o impacto combinado das variáveis meteorológicas sobre a morbidade/mortalidade é menos amplamente examinado ${ }^{(1,2)}$.

Além disso, há, muitas vezes, um atraso entre a morbidez/mortalidade e as condições meteorológicas, além do impacto imediato do tempo sobre a saúde, porque o efeito dos parâmetros de tempo pode ocorrer depois de certo atraso. No entanto, essa estrutura de defasagem entre o tempo e a morbidade/mortalidade não tem sido amplamente investigado $^{(3)}$.

Taxas elevadas de morbidade mostram a amplitude e a necessidade do estudo das doenças do aparelho respiratório (DAR), especialmente em crianças menores de cinco anos e em maiores que 60 anos de idade. A incidência das DAR é semelhante em todo o mundo, seja em países desenvolvidos ou em desenvolvimento; no entanto, existe diferença na tendência a maior frequência e na gravidade das infecções das vias aéreas, em especial as pneumonias. Isso acarreta aumento nas taxas de mortalidade em até trinta vezes nos países em desenvolvimento ${ }^{(4)}$.

Vários fatores estão associados no agravamento de DAR, como desnutrição, tabagismo passivo, escolaridade materna e densidade de moradores por domicílio ${ }^{(5)}$.

Destacam-se os fatores ambientais, como a poluição do ar e as variáveis climáticas, como determinantes para o aumento dos casos e da gravidade de DAR em menores de cinco anos de idade. Mudanças climáticas bruscas ajudam a piorar a qualidade do ar respirado, sobretudo quando a massa de ar frio dificulta a corrente de ventos e faz precipitar o material particulado da atmosfera nas grandes cidades. Com isso, há aumento significativo nos casos de pneumonia, asma e bronquite ${ }^{(6,7)}$.

Estudo realizado com crianças na região de São Paulo entre mortalidade por doenças respiratórias e a relação com umidade, temperatura e poluentes ambientais mostrou que houve uma associação significativa e um aumento de cerca de $20 \%$ no atendimento emergencial das doenças respiratórias $^{(8)}$.

Baseado no exposto, o presente trabalho tem por objetivo avaliar a relação entre variáveis meteorológicas e doenças respiratórias por faixa etária.

\section{MÉTODOS}

Este é um estudo ecológico de séries temporais, realizado no município de Campo Grande, Mato Grosso do Sul (MS), Brasil. A cidade de Campo Grande (S 20²6'16" W 5432'16" - 677 metros de altitude) localiza-se na região Centro-Oeste do Brasil. Encontra-se a cerca de $1.200 \mathrm{~km}$ ao sul da região sul da Bacia Amazônica e a cerca de 150 $\mathrm{km}$ a leste do Pantanal, maior planície inundável do mundo. Situa-se no planalto de Maracaju, divisor de águas entre as bacias hidrográficas do Rio Paraná e Rio Paraguai, e a cerca de $100 \mathrm{~km}$ a leste da Cordilheira dos Andes ${ }^{(9)}$.

Segundo a classificação climática de Köppen-Geiger, o clima da região de Campo Grande é classificado como tropical, com estação seca, caracterizado por temperaturas elevadas $\left(18^{\circ} \mathrm{C}\right.$ a $28^{\circ} \mathrm{C}$, com amplitude térmica de $5^{\circ} \mathrm{C}$ a $7^{\circ} \mathrm{C}$ ) e duas estações bem definidas, uma chuvosa e outra seca. Apresenta alto índice pluviométrico, em torno de 1500 $\mathrm{mm} / \mathrm{ano}$, com o período chuvoso começando em outubro e estendendo-se até abril, e o período seco correspondendo aos demais meses do ano ${ }^{(10)}$.

Obtiveram-se, na Secretaria Municipal da Saúde (SMS), os dados diários dos atendimentos ambulatoriais nas unidades de saúde do município, os quais se referem aos atendimentos de doenças respiratórias na faixa etária de 0 a 80 anos. O período analisado compreendeu os meses de janeiro de 2008 a dezembro de 2011. Utilizaram-se as bases de dados referentes ao Sistema de Informações Hospitalares do Sistema Único de Saúde (SUS), codificadas de acordo com a Classificação Internacional de Doenças (CID) - $10^{\mathrm{a}}$ Revisão (CID10 J10 a J18). Os dados analisados referemse ao Capítulo X da CID 10, que abrange as doenças do aparelho respiratório (DAR).

Informações sobre precipitação, temperatura do ar, umidade e velocidade dos ventos foram obtidas junto à Embrapa - Gado de Corte - Campo Grande (MS). 
Foram considerados como variável dependente $(\mathrm{Y})$ os coeficientes por doenças respiratórias; e como variável independente $(\mathrm{X})$, os anos de estudo. A transformação da variável "ano" na variável "ano-centralizado" (ano menos o ponto médio do período de estudo) fez-se necessária, já que, em modelos de regressão polinomial, os termos da equação frequentemente são altamente correlacionados, e expressar a variável independente como um desvio de sua média reduz substancialmente a autocorrelação entre eles.

Realizou-se a análise de tendência da série histórica utilizando-se um modelo de regressão linear múltipla que melhor descreveu a relação existente entre a variável independente $\mathrm{X}$ (precipitação, temperatura mínima e máxima, umidade relativa, velocidade dos ventos) e a variável dependente $\mathrm{Y}$ (coeficientes de hospitalização por DAR), segundo a equação $Y=\beta_{0}+\beta_{1} X_{1}+\beta_{2} X_{2}++\ldots+$ $\beta_{\mathrm{k}} \mathrm{X}_{\mathrm{k}}+\varepsilon$; em que k: número de variáveis; $\mathrm{X}_{\mathrm{j}}$ : regressores; $\beta_{\mathrm{j}}$ : estimadores; $\varepsilon$ : erro padrão. Como medida de precisão, utilizou-se o coeficiente de determinação $\left(\mathrm{R}^{2}\right)$. A análise dos resíduos confirmou a suposição de homocedasticidade do modelo $^{(11)}$.

Testes qui-quadrado com intervalo de confiança de 95\% para diferenças de proporções foram utilizados para a verificação de associações entre as covariáveis e a variávelresposta. Para variáveis contínuas e paramétricas, utilizouse o teste de ANOVA, e para variáveis não paramétricas, o teste de Kruskal-Wallis.

Como variável dependente para análise de regressão, coletou-se o total de casos de DAR (número de internações hospitalares) por faixa etária. Modelos de regressão linear múltipla incluíram todas as "variáveis-resposta" (contínuas) que se mostraram estatisticamente associadas à gravidade de DAR. Foram entendidos como testes de associação estatisticamente significantes valores de $p$ menores que 0,05; testes de distribuição de Fisher-Snedecor com valores críticos de $\mathrm{F}$, tais que $\left(\mathrm{F}>\mathrm{F}_{\mathrm{c}}\right)=0,05$; e coeficientes de explicação $\left(\mathrm{R}^{2}\right)$ que não incluíssem valores nulos.

Verificou-se a distribuição normal das diversas variáveis por intermédio do método de Kolmogorov-Sminov (KS), como também foram analisadas a heterocedasticidade e a inexistência de colinearidade, por meio da utilização de gráficos de plotagem e testes estatísticos apropriados. Nessa fase, optou-se pela suavização das variáveis, devido ao fato de elas terem apresentado distribuição assimétrica no período analisado. Desse modo, foi transformada em variável binária, do tipo presença/ausência (1/0), o que permitiu a sua utilização na modelagem da regressão linear múltipla. Também se realizou a modelização de categorias pela transformação em variáveis do tipo "dummy", utilizando os seus respectivos quartis, na tentativa de se encontrar pontos de corte que pudessem explicar melhor o modelo.
Para se verificar a inexistência de autocorrelação entre o escore de gravidade da DAR e o consequente pressuposto de independência dos erros, utilizou-se a análise gráfica dos resíduos de Student, pois a utilização do método de Durbin-Watson testa a hipótese que indica a inexistência da autocorrelação residual ter sido igual a zero, comprometida pelo pequeno tamanho da série analisada.

Quanto à análise residual, observou-se uma distribuição gaussiana de resíduos, aproximando-se de um padrão horizontal de dispersão em torno de sua média, que apresentou valor igual a zero $(0,0)$. A heterocedasticidade e a linearidade também puderam ser verificadas por meio de plotagem em gráficos de resíduos, apresentando distribuição aleatória, sem um comportamento cíclico ou de tendência definidos, os quais inviabilizariam o modelo de regressão.

Finalmente, na tentativa de se encontrar modelos que demonstrassem melhor o relacionamento entre variáveis ambientais e internação por DAR, explorou-se o comportamento das associações mediante a transformação curvilínea das variáveis-resposta, com consequente realização de regressão. No entanto, não se verificou diferença entre os níveis de significância e os coeficientes de explicação que inviabilizasse a utilização de regressão linear, uma vez que os pressupostos para a sua realização foram satisfeitos após os ajustes referidos anteriormente.

Utilização do Erro Quadrático Médio (EQM) para verificar a destreza do modelo:

$$
E Q M=\sqrt{\frac{1}{n}}\left\{\sum_{i=1}^{n}\left(p_{i}-O_{i}\right)^{2}\right\}
$$

Em que: Pi é o valor estimado e Oi, o valor observado. Em todas as análises, considerou-se o nível de significância de $5 \%$.

\section{RESULTADOS}

Ocorreram 12.067 internações por doenças respiratórias no período de investigação da presente pesquisa. Quando analisado o número de internações por doenças respiratórias por faixa etária, as maiores médias de internações ocorreram na de 1 a 4 anos, com média de $42,09 \%$ (5.079), e acima de 60 anos, com média de 27,14\% (3.275), sendo as pneumonias predominantes, com $81,0 \%$ (9.774) das internações. Verificou-se menor intensidade de internações em janeiro, e maior para junho e julho.

A Tabela 1 apresenta os valores dos riscos relativos ao modelo 1-4 anos e $>60$ anos e os coeficientes $b$ assumindo valores negativos e positivos, dependendo da variável, confirmando que o número de internações diminui quando aumentam esses valores; ou seja, quanto menor o índice de conforto térmico humano, maior o número de pessoas com doenças do aparelho respiratório ou vice-versa, e maior risco relativo ao intervalo de confiança de $95 \%$ que envolve 
Tabela I - Coeficientes de regressão, erro quadrático médio, risco relativo e coeficiente de correlação para os intervalos de confiança de 95\% para as faixas etárias estudadas. Campo Grande-MS, 2008-2011.

\begin{tabular}{cccccccccc}
\hline $\begin{array}{c}\text { Faixa etária } \\
\text { (anos) }\end{array}$ & $\mathbf{I}$ & $\boldsymbol{\beta 1}$ & $\boldsymbol{\beta 2}$ & $\boldsymbol{\beta 3}$ & $\boldsymbol{\beta 4}$ & $\boldsymbol{\beta 5}$ & EQM & $\mathbf{R R}$ & $\mathbf{R}^{\mathbf{2}}$ \\
\cline { 2 - 7 } & & lag 1 dia & lag 2 dias & lag 4 dias & lag 4 dias & lag 2 dias & & & \\
\hline $0-4$ & 603 & $-0,617$ & $-31,4$ & 29,3 & 13,1 & $-3,07$ & 13 & 1,98 & 88,10 \\
$5-9$ & 56 & $-0,053$ & $-8,25$ & 8,93 & 12,3 & $-0,68$ & 3,98 & 0,96 & 78,90 \\
$10-14$ & $-4,5$ & $-0,0048$ & $-0,46$ & 0,08 & 3,65 & 0,195 & 1,25 & 0,30 & 81,50 \\
$15-19$ & $-49,4$ & 0,0167 & 3,03 & $-3,47$ & 2,11 & 0,629 & 1,21 & 0,19 & 70,20 \\
$20-24$ & 24,7 & $-0,0006$ & $-1,21$ & 0,95 & 0,782 & $-0,043$ & 1,22 & 0,17 & 34,20 \\
$25-29$ & $-1,1$ & $-0,0131$ & $-0,31$ & 0,52 & 0,914 & 0,122 & 1,05 & 0,19 & 49,00 \\
$30-34$ & $-9,6$ & $-0,0041$ & 0,57 & $-0,49$ & 0,546 & 0,187 & 1,05 & 0,33 & 29,10 \\
$35-39$ & 48,7 & $-0,0408$ & $-4,08$ & 4,94 & 0,758 & $-0,517$ & 1,09 & 0,39 & 51,30 \\
$40-44$ & $-52,1$ & 0,0099 & 3,19 & $-3,27$ & 1,39 & 0,615 & 0,71 & 0,26 & 78,50 \\
$45-49$ & $-48,2$ & 0,0151 & 2,86 & $-2,84$ & 2,5 & 0,325 & 1,71 & 0,33 & 52,70 \\
$50-54$ & 9,2 & $-0,0043$ & $-1,01$ & 0,97 & 1,67 & 0,0067 & 1,41 & 0,41 & 47,00 \\
$55-59$ & -19 & $-0,001$ & 0,92 & $-0,9$ & 2,72 & 0,126 & 1,8 & 0,62 & 53,40 \\
$>60$ & 89 & $-0,118$ & $-3,1$ & 2,2 & 5,16 & 1,41 & 5,66 & 1,18 & 77,40 \\
\hline
\end{tabular}

I-intercepto $_{; \beta 1}$-precipitação $\beta_{2}$-temperatura máxima; $\beta_{3}$-temperatura mínima; $\beta_{4}$-velocidade dos ventos; $\beta_{5}$-umidade relativa do ar; EQMerro quadrático médio; RR-risco relativo; coeficiente de correlação $\mathrm{R}^{2}$.

temperaturas, umidade relativa, precipitação e velocidade dos ventos ${ }^{(12)}$.

No modelo final da regressão linear múltipla, certificou-se que a função linear, selecionada para o modelo de regressão, apresentou distribuição aleatória ao redor da linha zero e não apresentou "outliers" em sua distribuição residual, o que caracterizou uma homogeneidade de variância no ajuste, validando, portanto, a análise final.

Nesse grupo de modelos estatísticos, a variável dependente $\left(\mathrm{n}^{\circ}\right.$ de internações hospitalares) é um processo de contagem, ou seja, é uma variável quantitativa discreta, e as variáveis independentes são variáveis candidatas a explicar o comportamento da série ao longo do tempo. Como variáveis independentes, utilizaram as variáveis meteorológicas (temperaturas máximas (lag de 2 dias) e mínimas (lag de 4 dias), umidades (lag de 4 dias), velocidade dos ventos (lag de 2 dias) e precipitação (lag de 1 dia). As variáveis "dia da semana" e "feriado" foram utilizadas para controlar a sazonalidade de curta duração. Para controlar sazonalidade de longa duração, utilizou-se a variável "ano".

$\mathrm{Na}$ análise da presente pesquisa, mediante uso da regressão linear múltipla, vê-se que persiste a influência de DAR na faixa etária de 1 a 4 anos, com precipitação com correlação de $(-0,89)$, temperatura mínima com correlação de $(-0,79)$, temperatura máxima com correlação de $(-0,72)$ e a velocidade dos ventos com correlação de $(0,50)$, sendo considerada associada à gravidade da DAR. Já para as faixas etárias maiores que 60 anos, as correlações são significativas para a temperatura máxima $(-0,41)$ e a umidade do ar $(0,44)$. Cumpre dizer que o coeficiente de explicação do modelo foi de $88,1 \%$ para $0-4$ anos e maior que $77,4 \%$ para maiores que 60 anos, conforme Tabela 1. Para as outras faixas etárias, foram significativas a temperatura máxima (10-14 anos; $20-24$ anos $-0,45 ;-0,42)$ e a velocidade dos ventos $(5-9 ; 10$ 14 ; 15-19; 40-44; 45-49; 55-59 anos; 0,$82 ; 0,82 ; 0,61 ; 0,43$; $0,68$ e 0,70$)$. Mesmo após a suavização de irregularidades e a realização de regressão curvilinear, chegou-se a melhores coeficientes de explicação ou significância estatística. Por outro lado, sabe-se que medidas de exposição individuais ou até mesmo determinadas sazonalidades endêmicas e possibilidades de variações irregulares, como epidemias, poderiam explicar os outros 12 e $23 \%$ de variabilidade não explicada pelo modelo.

\section{DISCUSSÃO}

Associações positivas foram encontradas no presente estudo entre as variáveis meteorológicas e as faixas etárias 
por doenças respiratórias em Campo Grande-MS. Esses efeitos são semelhantes aos encontrados em outras cidades do Brasil, em especial àqueles observados na cidade de São Paulo $^{(13)}$, tanto na diversidade das variabilidades dos valores meteorológicos associados quanto na magnitude dos efeitos estimados. $\mathrm{O}$ aumento das internações por doenças respiratórias nos períodos mais frios do ano deve-se às baixas temperaturas, o que mostra que os riscos relativos por internações por doenças respiratórias estão associados às faixas etárias de $0 \mathrm{a} 4$ anos e $>60$ anos.

Pela análise dos dados coletados na presente pesquisa, os resultados mostram que foi marcante a frequência da DAR em menores de quatro anos durante os anos de estudo, pois quase a metade $(42,09 \%)$ dos atendimentos dessa faixa etária se deve a crianças com sinais e sintomas decorrentes de algum diagnóstico de DAR, ficando todas as outras faixas etárias com a outra metade. Isso é o dobro do encontrado em outra investigação ${ }^{(13)}$, que estudou crianças da mesma faixa etária no serviço de pronto atendimento de pediatria e encontrou prevalência de $25,6 \%{ }^{(13)}$.

Acredita-se que a temperatura afeta a pressão arterial e a viscosidade do sangue ${ }^{(14,15)}$. A pressão sanguínea aumenta com a temperatura mais baixa, embora a temperatura possa aumentar no período noturno ${ }^{(16)}$. A baixa temperatura também aumenta a viscosidade do sangue e da frequência cardíaca $^{(14)}$ e pode desencadear doenças do aparelho circulatório.

Em geral, a temperatura mínima pode provocar doenças respiratórias e broncoconstrição, aumentando a susceptibilidade de infecção pulmonar, induzida pela inalação de ar frio. Na exposição ao calor, o sangue é forçado para dentro da periferia, para promover a perda de calor e, portanto, aumentar a pressão do sangue, porque os vasos sanguíneos perto do centro do corpo são constringidos ${ }^{(17)}$. Além disso, a viscosidade do sangue pode aumentar com altas temperaturas ${ }^{(18)}$, e esses aumentos vão interagir com fibrilação atrial, provocando coágulo sanguíneo. Além das diferenças de gênero nas respostas termorreguladoras ao calor, o tempo de baixa tolerância ao calor, devido à acumulação excessiva de calor do corpo ${ }^{(19)}$, contribui para a maior susceptibilidade à doença respiratória.

O tempo-morbidade está associado na faixa etária $\geq 60$ e estabelece que os idosos são mais suscetíveis ao estresse tempo. Essa relação tempo-morbidade é evidente, devido à falha do mecanismo de defesa homeostático com o avanço da idade, que por sua vez provocaria dificuldades respiratórias ou outras doenças ${ }^{(20)}$.

A DAR está entre as principais causas de consulta médica, sendo responsável por mais de um terço delas e por um grande número de hospitalizações em crianças e adultos, na maioria dos países ${ }^{(21)}$. A faixa etária com maior incidência de DAR ocorre entre seis meses e quatros anos e maiores que 60 anos de idade, tanto em países desenvolvidos quanto naqueles em desenvolvimento. A diferença da morbidade da DAR entre os países mais ricos e os em desenvolvimento é que nestes são mais frequentes os casos mais graves, particularmente as pneumonias, as quais muitas vezes vêm com complicações fatais. É fato descrito que a taxa de mortalidade da DAR é maior nos países com menor qualidade de vida dos seus habitantes ${ }^{(21)}$.

Outro indicador de gravidade da DAR é a taxa de hospitalização em determinado serviço. Sabe-se que as complicações da DAR geralmente necessitam de atendimento hospitalar, sendo as infecções das vias aéreas inferiores, sobretudo as pneumonias e as broncopneumonias, as que mais requerem hospitalizações, em razão de sua gravidade, podendo chegar ao óbito. Comparando todas as internações de crianças menores de cinco anos, por qualquer diagnóstico, os dados da OPAS/OMS ${ }^{(22)}$ mostram que as pneumonias são responsáveis por $20 \%$ a $40 \%$ das hospitalizações nos países em desenvolvimento, e as frequentes hospitalizações poderiam ser atribuídas a formas que poderiam ser eficazmente tratadas em ambulatório. No atual estudo, em Campo Grande-MS, a pneumonia em lactentes, crianças e adultos é responsável por $81 \%$ do número de internações por DAR. O maior número de casos graves pode estar relacionado à maior prevalência de diferentes fatores associados que agravam a DAR, como: desnutrição, desmame precoce, consulta tardia e inadequada avaliação e classificação dos casos pelo pessoal de saúde, quando da primeira consulta.

Sabe-se que fatores ambientais influenciam na prevalência e no perfil de gravidade da DAR, havendo aumento da demanda ambulatorial nos meses de inverno, principalmente dos quadros benignos de infecções das vias aéreas superiores. Contudo, as pneumonias e as bronquiolites têm significativo aumento proporcional, fato que reforça a demanda hospitalar ${ }^{(7)}$.

Em Campo Grande-MS, no período seco, a umidade relativa do ar chega a níveis incompatíveis com a integridade das vias aéreas ${ }^{(10)}$. Sabe-se que, para o bom funcionamento das vias aéreas, há necessidade de certo grau de umidade, que não deverá ser inferior a $60 \%{ }^{(23)}$. É grande o trabalho que a via aérea tem para tentar manter a homeostase interna com a qualidade do ar respirado, pois a umidade relativa do ar mínima é muito baixa, em torno de $19 \%$ a $27 \%$ durante o período $\operatorname{seco}^{(23)}$.

Os resultados apresentados no estudo em questão representam uma aproximação quantitativa do impacto dos dados climáticos na saúde da população. É importante salientar que o desfecho aqui estudado - a hospitalização - é apenas um dos muitos efeitos causados pelos dados 
meteorológicos. Efeitos como ocorrência de sintomas, uso de medicação, condições sociais, redução na atividade física, entre outros, não foram avaliados no presente trabalho. Estes, considerados de menor gravidade para a saúde do indivíduo, são de grande importância para a saúde pública, dada a elevada frequência com que ocorrem e devido ao impacto negativo na qualidade de vida das pessoas e de consequências econômicas negativas, como o absenteísmo escolar e no trabalho.

A Organização Mundial da Saúde (OMS) e o Programa das Nações Unidas para o Meio Ambiente (PNUMA) avaliam que as condições de conforto térmico afetarão milhares de pessoas, uma vez que estão relacionadas ao clima e à forma de apropriação e interação com os espaços locais e/ou regionais. Em geral, populações carentes sofrem mais com o calor excessivo e têm dificuldades de adaptação às novas condições, em virtude da falta de recursos financeiros e, consequentemente, das condições de moradia. Também as áreas urbanas são mais afetadas que as rurais, sobretudo por causa da abundância de superfícies que retêm o calor. Desse modo, o aumento e/ou a diminuição de temperatura afetará a saúde humana de diversas maneiras e em diferentes proporções. Segundo o IPCC (Intergovernmental Panel on Climate Change), além das mortes promovidas diretamente pela ocorrência de um evento extremo e dos impactos desse evento na infraestrutura local e nos recursos naturais, as variabilidades climáticas podem levar a um aumento da subnutrição, à disseminação de doenças transmitidas pela água ou por outros vetores, ao aumento da frequência de doenças cardiorrespiratórias pela poluição atmosférica, à redução da produtividade da população afetada e ao aumento dos gastos com medicamentos e cuidados com a saúde. Nas últimas três décadas, problemas de ordem social, acrescidos de mudanças na temperatura, umidade do ar e na biodiversidade, alteraram o equilíbrio entre predadores, competidores e presas, impedindo que pestes e patógenos fiquem sob controle, o que contribui para o ressurgimento de doenças infecciosas ${ }^{(24)}$.

Estimar o risco para a saúde da população em razão dos dados climáticos é um primeiro passo para o planejamento e a implementação de ações visando a um ambiente mais saudável. A produção de dados tecnicamente bem embasados é fundamental para a formulação de políticas públicas e tomada de decisões que promovam o desenvolvimento socioeconômico e levem em conta questões ambientais e a qualidade de vida das pessoas. Os resultados aqui apresentados indicam, em termos quantitativos, o risco para um aumento no número de hospitalizações de crianças menores que 4 anos e idosos maiores que 60 anos, de acordo com um aumento ou uma diminuição de temperaturas, umidades, precipitação e velocidade dos ventos na cidade de Campo Grande-MS. Espera-se que os dados apresentados sirvam como ferramentas para um melhor entendimento do impacto das variáveis meteorológicas na saúde das pessoas, colaborando para o monitoramento e saneamento dessa questão em Campo Grande-MS e em outras metrópoles.

\section{CONCLUSÃO}

Este estudo teve a pretensão de construir um modelo preditor que pudesse estimar o número de internação por doenças do aparelho respiratório por faixa etária e demonstrar a associação entre esse desfecho e as principais variáveis ambientais em Campo Grande-MS, levandose em conta a relevância de se conhecer as características epidemiológicas da doença frente às peculiaridades climáticas da região. A utilização de regressão linear múltipla foi satisfatória para a demonstração da associação entre internação por doenças do aparelho respiratório (DAR) por faixa etária e as variáveis de estudo. Conclui-se, com base nas investigações realizadas, que há correlação significativa entre os parâmetros climáticos e as internações por doenças respiratórias.

\section{REFERÊNCIAS}

1. Baccine M, Biggeri A, Accetta G, Kosatsky T, Katsouyamni K, Analitis A, et al. Heat effects on mortality in 15 European cities. Epidemiology. 2008;19(5):711-9.

2. Jimenez-conde $\mathrm{J}$, Ois A, Rodriguez-campello A, Cuadrado-godia E, Subirana I, ROQUER J: Weather as a trigger of stroke. Cerebrovasc Dis. 2008;26(4): $348-54$.

3. Souza A, Guo Y, Pavão HG, Fernandes WA. Effects of Air Pollution on Disease Respiratory: Structures Lag. Health. 2014;6(12):1333-9.

4. Oliveira BRG, Viera CS, Furtado MC, Mello DF, Lima, RAG. Perfil de morbidade de crianças hospitalizadas em um hospital público: implicações para a Enfermagem. Rev Bras Enferm. 2012;65(4):586-93.

5. Islam F, Sarma R, Debroy A, Kar S, Pal R. Profiling Acute Respiratory Tract Infections in Children from Assam, India. J Glob Infect Dis. 2013;5(1):8-14.

6. Rosa AM, Ignotti E, Botelho C, Castro HA de, Hacon SS. Doença respiratória e sazonalidade climática em menores de 15 anos em um município da Amazônia brasileira. J Pediatr (Rio J.). 2008;84(6):543-9.

7. Silva Júnior JL, Padilha TF, Rezende JE, Rabelo EC, Ferreira AC, Rabahi MF. Effect of seasonality on the occurrence of respiratory symptoms in 
a Brazilian city with a tropical climate. J Bras Pneumol. 2011;37(6):759-67.

8. Arbex MA, Santos UP, Martins LC, Saldiva PHN, Pereira LAA, Braga ALF. A poluição do ar e o sistema respiratório. J Bras Pneumol. 2012;38(5):643-655.

9. Souza A, Aristone F, Pavão HG, Fernandes WA. Development of a Short-Term Ozone Prediction Tool in Campo Grande-MS-Brazil Area Based on Meteorological Variables. OJAP. 2014;3(2):42-51.

10 Souza A, Pavao HG, Lastoria G, Gabas SG, Paranhos Filho AC, Cavazzana GH. Distribuição espacial da relação precipitação/número de dias de chuvas em Campo Grande-MS. Seminário de recursos hídricos da bacia hidrográfica do Paraíba do Sul. Taubaté, 2009.

11. Latorre MRDO, Cardoso MRA. Análise de séries temporais em epidemiologia: uma introdução sobre aspectos metodológicos. Rev Bras Epidemiol. 2001;4(3):145-52.

12. Souza A, Schujmann E, Fachel JMG, Fernandes, WA. Indicadores ambientais e doenças respiratórias em crianças. Mercator. 2013;12(27):101-9.

13. Silva AMC, Matos IE, Freitas SR, Longo KM, Hacon SS. "Material particulado (PM2. 5) de queima de biomassa e doenças respiratórias no sul da Amazônia brasileira; Particulate matter (PM2. 5) of biomass burning emissions and respiratory diseases in the south of the Brazilian Amazon." Rev Bras Epidemiol. 2010;13(2):337-51.

14. Rosa AM, Ignotti E, Botelho C, Castro HA, Hacon SS. Respiratory disease and climatic seasonality in children under 15 years old in a town in the Brazilian Amazon. J Pediatr (Rio J). 2008;84(6):543-9.

15. Kunst AE, Looman CW, Mackenbach JP. Outdoor air temperature and mortality in the Netherlands: a time series analysis. Am J Epidemiol. 1993;137(3):331-41.

16. Mercer JB, OSterud B, Tveita T. The effect of short-term mild cold exposure on risk factors for cardiovascular disease. Thromb Res. 1999;95(2):93-104.

17. Jehn M, Appel LJ, Sacks FM, Miller ER. The effect of ambient temperature and barometric pressure on ambulatory blood pressure variability. Am J Hypertens. 2002; 15(11):941-5.

18. Burse RL: Sex differences in human thermoregulatory response to heat and cold stress. Human factors. 1979;21(6):687-699.
19. Keatinge WR, Cloeshaw SRK, Easton JC, Cotter F, Mattock MB, Chelliah R. Increased platelet and red cell counts, blood viscosity and plasma cholesterol level during heat stress and mortality from coronary and cerebral thrombosis. Am J Med. 1986; 81(5): 795-800.

20. Shirreffs SM: Heat stress, thermoregualtion and fluid balance in women. Br J Sports Med. 1999;33(4):225.

21. Pan WH, Li LA, Tsai MJ. Temperature extremes and mortality from coronary heart disease and cerebral infraction in elderly Chinese. Lancet. 1995;345(8946):353-5.

22. Niobey FML, Duchiade MP, Vasconcelos AGG, Carvalho ML, Leal MC, Valente JC. Fatores de risco para morte por pneumonia em menores de um ano em região metropolitana do Sudeste do Brasil. Um estudo de tipo caso-controle. Rev Saúde Pública. 1992;26(4):229-38.

23. Organizacion Panamericana de la Salud, Organizacion Mundial de la Salud. Investigaciones operativas prioritarias para evaluar el impacto de las acciones de controli de las infecciones respiratorias agudas. Genebra: OPS/OMS; 1992.

24. Hungria H, Palombini BC, Pereira AP. Vias respiratórias superiores e inferiores - Correlações fisiopatológicas e clínicas. In: Bethlem N, organizador. Pneumologia. $4^{\mathrm{a}}$ ed. São Paulo: Atheneu; 1996. p. 69-76.

25. Intergovernmental Panel on Climate Change - IPCC. Summary for policymakers. In: Solomon D, Qin M, Manning Z, Chen M, Marquis KB, Averyt M, editors. Climate change 2007: The physical science basis. Contribution of Working Group I to the Fourth Assessment Report of the Intergovernmental Panel on Climate Change. Cambridge: Cambridge University Press, 2007. [2014 Feb 03]. Available in: http://ipccwg1.ucar.edu/wg1/Report/AR4WG1_Print_SPM.pdf

\section{Endereço para correspondência:}

Amaury de Souza

Universidade Federal de Mato Grosso do Sul

Departamento de Física

Cidade Universitaria, $\mathrm{s} / \mathrm{n}^{\mathrm{o}}$

Bairro: Universitário

CEP: 79070-900 - Campo Grande - MS - Brasil

E-mail: amaury.de@uol.com.br 